Volume 3, Issue 1, 2016

\title{
Carcinoma Right Breast (post-operative Chemotherapy and Radiotherapy) with bone and lung metastases: A Case Report and Review of Literature
}

\author{
Article by Kavita Gupta ${ }^{1}$ \\ PhD Scholar at Texila American University, Guyana \\ E-mail: 16kavitagupta@texilaconnect.com
}

\begin{abstract}
It is generally observed that triple negative breast cancer patients do not get benefited from endocrine targeted therapies. However, by a thorough Systematic review and critical analysis of the literature it could be depicted that a subset with Androgen Receptor expression could respond to anti-androgen therapies up to some extent. Presented here is a 65-years-old Postmenopausal female with a 5-year history of Carcinoma Right Breast (postoperative Chemotherapy and Radiotherapy) with bone and lung metastases, advanced, Grade III tumor diagnosed as Metastatic Adenocarcinoma on histology. This case had been presented to identify the role of Androgen Receptors in inhibiting and suppressing the tumor growth in breast oncology and to conclude that traditional Anti-Androgen therapy, that is, Bicalutamide could be considered as a differential diagnosis of Triple negative breast cancer metastatic tumors. Therefore, the present article represents some deeper insights regarding Androgen, Androgen-Receptor, Anti-Androgen drug-Bicalutamide and their role of action in the post-menopausal Triple Negative Breast cancer women with positive expression of Androgen Receptor.
\end{abstract}

Keywords: Breast, Lungs, Carcinoma, Metastasis, Bicalutamide, Androgen Receptor, Post-menopausal, Androgen, Estrogen, HER2, TNBC.

\section{Abbreviations}

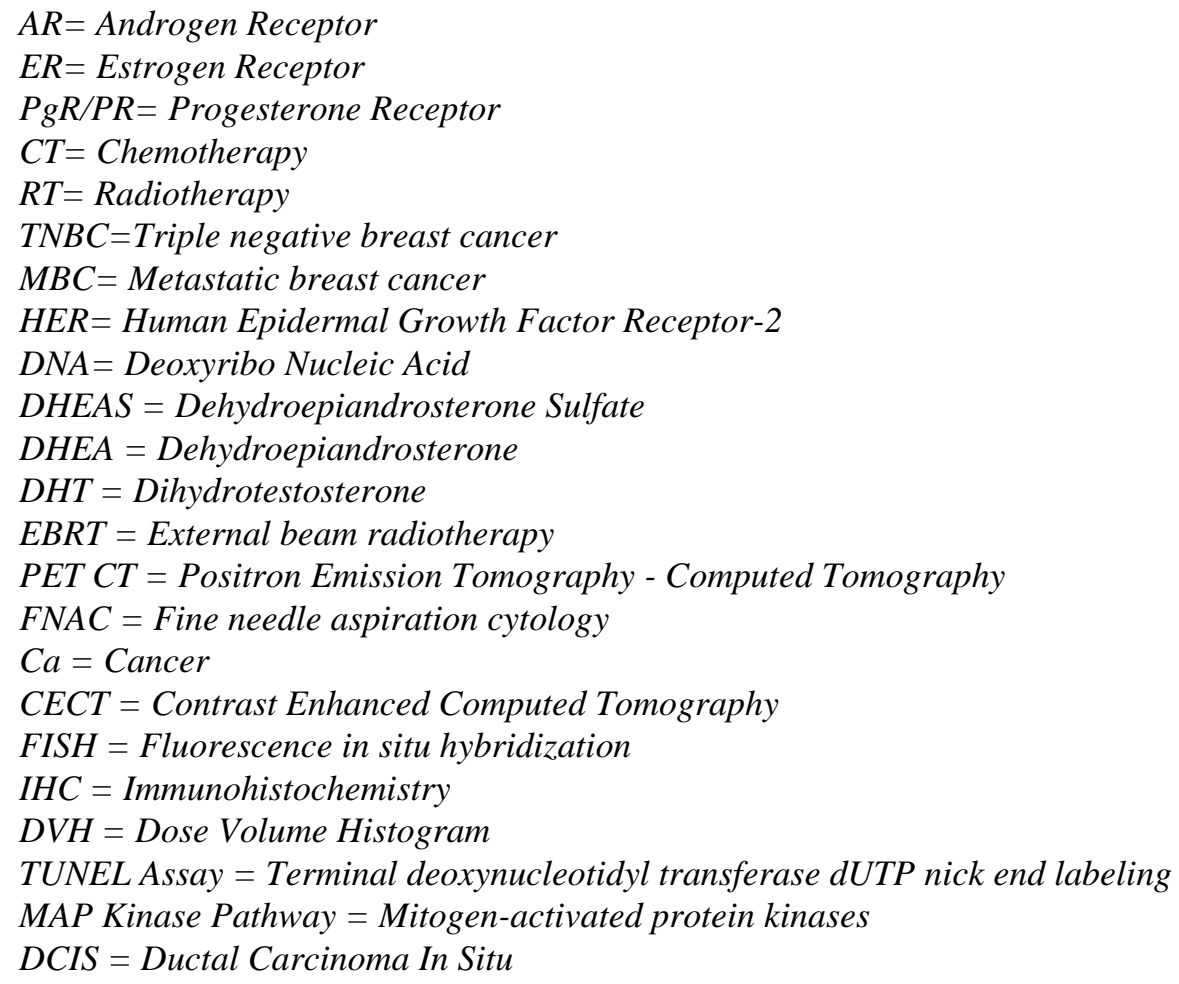


South American Journal of Clinical Research

Volume 3, Issue 1, 2016

\section{Introduction}

Triple Negative Breast Cancer is considered to be the most diverse group of cancers and therefore subtyping is highly essential for the identification of better molecular basedtherapies [6]. From the various preclinical and clinical trials' study it had been elucidated that due to lack of ER and PR expression in TNBC patient, HER2 amplification might led to heterogeneity of the underlying disease and absence of well-defined molecular targets on the other hand [6]. TNBC tumors were generally characterized to be larger in size, of high grade, involving lymph node at diagnosis and are biologically the most aggressive form of carcinoma [6].

According to the hypothesized principle it is being stated that breast cancer might be a result of hormonal imbalance of Estrogens conversion to Androgens [3]. Estrogen was considered to be the dominant female sex hormone which was then targeted for the anti-breast cancer therapy [3]. The Estrogen Receptor (ER) had been predicted to regulate the growth and differentiation of the normal mammary gland and was considered to be important in the development and progression of about $70 \%$ of breast cancer [2]. The Androgen Receptor was found to be positive in approximately $80 \%$ of the breast cancer patients [10].

According to Rakha, E.A and El-Sayed, M.E, et al. studies, previous DNA microarray and Immunohistochemistry analyses had shown that $80 \%$ to $90 \%$ of Triple Negative tumors were found to be basal like [9]. It was more likely that Triple negative tumors differentiated poorly as they got associated with a higher rate of recurrence or relapse thereby, reducing the overall survival of the patient suffering [9]. It would be appropriate to state that Androgen Receptor expression could be considered as a positive prognostic factor in such cases [5]. Going through the various epidemiological studies it can be inferred that there was some correlation between high endogenous androgens and breast cancer risk [5]. Before starting the discussion, it was highly essential to understand the terms Androgen, Androgen Receptor, and their mechanism of action in brief [5].

The Androgen receptor gene was defined to be located on the X-chromosome [5]. It was classified as a member of Steroid hormone receptor family [5]. When the Androgen bounds to cytosolic Androgen Receptor, the androgen receptor underwent a conformational change that was, dimerization and phosphorylation followed by interaction with androgen response elements in the promoter region of androgen dependent genes, and then finally entered the nucleus [5].

Principally, there were 5 androgens found in women discovered up till date, namely, Dehydroepiandrosterone Sulfate (DHEAS), Dehydroepiandrosterone (DHEA), Androstenedione, Testosterone, and Dihydrotestosterone (DHT) [5].

The above listed hormones were expected to be present in pre-menopausal women [5]. However, studies support the fact that after menopause there was a marked substantial decrease in circulating levels of Estrogen, Progesterone, Testosterone, Dehydroepiandrosterone Sulfate, Dehydroepiandrosterone Androstenedione declination by $50 \%$ which further stated that it might be because of post-menopausal ovary actively start producing and synthesizing Androgens [5]. In Post-menopausal women, adipose tissue was found to be the primary source of endogenous Estrogen through their aromatization to estradiol and estrone (primary form of Estrogen in post-menopausal women) in the breast [5]. There is relevant evidence from different prospective studies, according to which it can be elucidated that with the increase in level of circulating androgens there was a relative higher risk of development of breast cancer in post-menopausal women [5]. Extensive literature review proposed that breast cancer risk can be developed either directly if Androgen increased cellular growth and proliferation via Androgen Receptor, or, indirectly by aromatization of Androgens to Estrogens [5].

According to Grattarola proposed 'Hyperandrogenic theory' in 1960s, Breast cancer patients often had elevated Androgen levels, increased urinary androgen secretion, atypical endometrial hyperplasia that ultimately led to increased luteal progesterone reduction [5]. Further in his theory, he also stated that hyperplasia of interstitial cell led to increased ovarian 
androgen production [5]. Later on, Secreto et al. proposed the 'Androgen Excess Theory' according to which increased level of circulating Androgens were correlated with an increased risk of development of breast cancer [5].

According to Key et al. pooled studies in post-menopausal women indicated an increased risk of breast cancer with increased level of sex hormones that included, Dehydroepiandrosterone Sulfate, (DHEAS) Dehydroepiandrosterone (DHEA), Androstenedione, Testosterone, and Dihydrotestosterone (DHT) [5]. However, on the other hand Missmer et al. reported no correlation between the Progesterone level and breast cancer risk [5].

The second aspect of the study was an anti-Androgen Receptor drug, Bicalutamide. Bicalutamide had been found to be poor water soluble drug that was initially diagnosed in the year 1995 for the treatment of advanced Prostate cancer in men [12]. The poor water solubility of Bicalutamide was associated with slow drug absorption which ultimately led to inadequate and variable bioavailability [12]. The empirical formula of Bicalutamide is: $\mathrm{C}_{18} \mathrm{H}_{14} \mathrm{~F}_{4} \mathrm{~N}_{2} \mathrm{O}_{4} \mathrm{~S}$ [12]. Taking into account, Bicalutamide is being classified as non-steroidal antiandrogens available in the form of oral tablets which acted mechanically by blocking the effect of Androgen that further inhibited the spread of cancerous cells [13]. The possible side effects associated with the use of Bicalutamide were: Nausea, Fatigue, hot flashes, limb edema, aspartate aminotransferase elevation, alkaline aminotransferase elevation, hyperbilirubinemia, alkaline phosphatase elevation, headache, backache, constipation, limb pain, anorexia, vaginal dryness, etc. [4].

\section{Presentation of the case report}

Sixty five years old female was diagnosed as a known case of carcinoma right breast in the month of February 2010. She underwent surgery followed by 6 cycles of Chemotherapy followed by EBRT up till September 2010. On follow up she developed lung and bone metastases in 2014. For which she received multiple cycles of palliative chemotherapy. PET CT was done on 18/05/15 and it was suggestive of right parasternal mass with skeletal erosion and right upper lobe of lung nodule. She was referred for Radiotherapy.

On 15/09/14, CT Chest was performed in which 2.4 X $1.2 \mathrm{~cm}$ nodule was seen in anterior segment of right upper lobe. Moreover, Lytic lesion with soft tissue component was seen at the right 4 th costosternal junction along with mild nodular pleural thickening seen in the right lung. This became the evidence for performing FNAC from chest wall lesion.

On 19/09/14, CT guided Biopsy from Right 4th Rib deposit was performed in which multiple linear core largest measuring $1.3 \mathrm{~cm}$ was examined. The section showed Foci of neoplastic cells in Glandular pattern. Cells had high N/C ratio, granular chromatin and cosspicuous nucleoli. Thus, Metastatic Adenocarcinoma in a known case of Ca Breast was confirmed.

On 18/05/15, PET was performed. The main purpose of performing PET scan was to evaluate the response in a recurrent $\mathrm{Ca}$ Right Breast who underwent oral chemotherapy Post 6-cycles of Chemotherapy. During PET scan 296-370 MBq 18F-FDG was administered I.V. and images were taken after 1 hour from skull base to mid-thigh. CT scan was done for attenuation correction and localization. On diagnosis metabolically active right parasternal soft tissue lesion was seen in right 3rd intercostal space which involved 3rd costal cartilage $(3.2 \times 1.9 \mathrm{~cm}$, SUV max 9.3). Moreover, mild periarticular tracer uptake was seen on the right shoulder joint, likely inflammatory. On the other hand, Right lung upper lobe showed a sub $\mathrm{cm}$ parenchymal nodule $(2.9 \times 0.9 \mathrm{~cm}$ ) with mild tracer uptake (SUV max 2.2). Sub cm lymph nodes with mild tracer uptake were also seen in right Para tracheal, prevascular, sub cranial and bilateral hilar regions, likely inflammatory.

This scan when compared with the patient's earlier study of February 2015, it was observed that right chest wall lesion showed increase in metabolic activity. Right lung upper lobe nodule also showed mild increase in the metabolic activity. 
South American Journal of Clinical Research

Volume 3, Issue 1, 2016

On 28/07/15, CECT CHEST was performed. Borderline sized lymph nodes were seen in the right lower peritracheal region sub cranial region. Ill-defined sub centimeter sized nodular infiltrate was also seen in apical segment of the right lung. Patchy small parenchymal infiltration was seen in basal segment of the right lung parenchyma in sub pleural location. Mild left fissured thickening was also seen.

On the other hand, Ill-defined soft tissue thickening with stranding in overlying subcutaneous fat was seen in the anterior chest wall in right parasternal location at the level of 3rd coastal cartilage.

Areas of coarse calcification were seen in rest of the right anterolateral chest wall, likely post-operative changes.

As compared to CT PET previous scan dated 18/05/15, right parasternal soft tissue thickening and right lung nodule showed mild resolution.

On 26/08/15, Review of Slide was done. $3 \mathrm{H} \& \mathrm{E}$ slides and 3 Paraffin blocks labelled as $\mathrm{B} / 1119 \mathrm{H} 10, \mathrm{~B} / 1119 \mathrm{~B} / 10, \mathrm{~B} / 1119 \mathrm{C} / 10$ were received. Two $\mathrm{H}$ \& $\mathrm{E}$ slides labelled as B/1119 $\mathrm{B} / 10$ and $\mathrm{C} / 10$ and sections from the corresponding blocks showed breast tissue with an invasive tumor composed of interconnecting masses and nests of pleomorphic malignant epithelial cells with moderate eosinophilic cytoplasm, well- defined cytoplasmic borders and enlarged moderate to markedly pleomorphic nucleus with irregular nuclear membrane, clumped chromatin and prominent nucleolus. Mitoses up to $6 / 10 \mathrm{hpf}$ seen (Field diameter: $0.44 \mathrm{~mm}$ ). Multifocal intratumoral necrosis was seen. Slide B/1119 H/10 and section from the corresponding block showed three lymph nodes with metastatic tumor deposits identified in one lymph node (1/3). Finally, Immunohistochemistry test diagnosed the Estrogen Receptor to be Negative for Estrogen receptors Immunomarker, and Progesterone Receptor to be Negative for Progesterone receptors Immunomarker. Herein, it was evaluated that Her2/neu: Score 2+; Equivocal, Weak circumferential membrane staining $>10 \%$ of cancer cells but the membrane staining ring was found to be thin (Score 2+). On the other hand, Ki 67: Proliferative index was found to be $15 \%$.

Final diagnosis: Slides and blocks for review (Breast, Right); Invasive ductal carcinoma, NOS; Nottingham Histologic score of $8(3+3+2)$; overall Grade III.

On 31/08/15, Her-2/neu by FISH (Fluorescence in situ hybridization) test was performed in which Total no. of cells scored were112; Total no. of HER2 signals were: 238; Total no. of CEP17 signals were: 280; Average HER2 signals/cells were: 2.12. Therefore, the Computed Ratio calculated was: 0.85 . These findings were indicative that the patient was not eligible for anti-HER2 therapy (Trastuzumab).

On 12/11/15, IHC (Immunohistochemistry 1 Marker) test was performed. Slides and Blocks of Right Breast were reviewed. According to Previous histopathology report (review), it was observed that the tumor was Invasive ductal carcinoma, Grade III, with ER and PgR status: Negative and Her2Neu score 2+: Equivocal. Androgen Receptor: 15\% of tumor cells were found to be weak positive.

\section{Treatment}

She was planned for Radiotherapy. The simulation was carried on with CT scan. The tumor volume and the critical structures were adequately contoured for dose calculation purpose. Treatment planning was performed on Multiplan as per the prescribed dosage, and dose prescription parameters were compiled with dose volume histogram. An optimized inverse treatment plan was created, such that $80 \%$ of isodose lines contour, while minimizing the dose to critical structures. DVH was approved and she received validation to a total dose of $24 \mathrm{~Gy}$ in 3 fractions, i.e., $800 \mathrm{cGy}$ per fraction to the right parasternal mass from 6/06/15 to 9/06/15 by 6 MV Photons and $76 \%$ of isodose lines contour of right lung lesion received 20 Gy in single fraction on 5/06/15 by 6 MV Photon. Treatment was carried out in the presence of Radiation Oncologist. Patient tolerated the treatment well.

Presently, on Oral Chemotherapy drug CALUTIDE (BICALUTAMIDE). 


\section{Discussion}

Evidence1: In a study performed by Cochrane, D.R., et al. the protein ratio of Androgen Receptor to Estrogen Receptor was examined in primary breast cancer in response to endocrine therapy [1]. Out of 2,171 invasive breast cancers in women enrolled in the Nurses' Health Study, 32\% of Triple Negative Breast Cancer was found to be positive for Androgen Receptor expression, which was confirmed by IHC diagnosis (Immunohistochemistry) [1]. It was concluded in the study that a subset of ER (-) breast cancers (molecular apocrine or luminal androgen receptor) retained Androgen Receptor that contain a gene expression pattern similar to that of ER $(+)$ breast cancers [1].

Evidence 2: In a study performed by Cochrane, D.R., et al., it was elucidated that the traditional anti-androgen Bicalutamide enhanced upregulation of SDF-1 and other E2regulated genes that led to the enhancement of E2-mediated breast cancer cell proliferation [1]. When Androgen Receptor bound to Bicalutamide, then these Androgen Receptors translocated to the nucleus and bound to the DNA of the cancerous cells thereby inhibiting the growth of molecular apocrine cell lines in vitro and in vivo, which was in support with the hypothesis that anti-androgen therapy could be successful in the treatment of TNBC tumors [1].

Evidence 3: In a study performed by Niemeier, LA., et al. the antitumor activity in targeting Androgen Receptor was studied in vivo in an MDA-MB-453 Xenograft model [8]. They performed an experiment in which MDA-MB-453 cells were implanted in the mammary gland of NOD/SCID mice [8]. The group of the mice that received the concomitant implantation of a DHT slow-release pellet, DHT dramatically promoted the growth of MDAMB-453 tumors in vivo $(\mathrm{P}=0.0003)$ [8]. Once the tumors attained a volume of $400 \mathrm{~mm}^{3}$, daily oral administration of Bicalutamide at $10 \mathrm{mg} / \mathrm{kg}$ was delivered to $50 \%$ of the mice carrying DHT pellets $(n=8)$ [8]. As a result, the treatment with Bicalutamide resulted in a significant inhibition of DHT-stimulated growth of MDA-MB-453 xenograft tumors $(\mathrm{P}=0.0007)$ and tumors regressed to control levels after 3 weeks of treatment [8]. Moreover, it was also observed through TUNEL assay that Bicalutamide treatment led to the substantial reduction in HER3 and p-AKT expression that eventually led to enhanced cancerous cell death [8]. When taken together these two observations, it can be concluded that Bicalutamide blocked androgen-stimulated oncogenic HER2/HER3 signal expression and inhibited the growth of ER (-)/HER2 (+)/ AR (+) breast tumors in vivo [8].

Evidence 4: In a study performed, it was observed that ER and HER2, mRNA expression had some correlation with IHC and FISH analyses [6].

Evidence 5: In a study performed by Shibahara, Y., et al., it was elucidated that the primary breast tumor consisted of androgen metabolizing enzymes, namely, 17 $\beta$ hydroxysteroid dehydrogenase type 5 (17 $\beta$ HSD5) and $5 \alpha$-reductase 1 (5 $\alpha 1)$ [10]. However, $17 \beta$ HSD5 and $5 \alpha 1$ immunoreactivity was decreased in metastatic lymph nodes [10]. $5 \alpha 1$ was found to be associated with significantly lower Ki-67 index [10]. On the other hand, double positivity of AR $5 \alpha 1$ expressed in lymph nodes was found to be associated with larger lymph node metastasis and higher Triple Negative Metastatic stage [10].

Evidence 6: In a study performed by Luo, X., et al., it was elucidated that Androgen Receptor was involved in the pathogenesis of breast carcinoma [7]. In their experiment, they performed IHC assays in order to, determine the expression of AR in 137 cases of triple negative breast cancer [7]. As a result, it was observed that the positive rate of AR was significantly lower in case of Triple Negative Breast Cancer patients $\left(27.7 \%\right.$, $\mathrm{Chi}^{2}=83.963$, $\mathrm{P}<0.001)$ [7]. Moreover, it was found that $\mathrm{AR}$ expression was somehow correlated with the menorrheal status of TNBC females $\left(\mathrm{Chi}^{2}=6.803, \mathrm{P}=0.023\right)$, node status $\left(\mathrm{Chi}^{2}=7.787, \mathrm{P}=\right.$ $0.005)$, with a 5 -year disease free survival $\left(\mathrm{Chi}^{2}=5.012, \mathrm{P}=0.025\right)$ and 5 -year overall survival $\left(\mathrm{Chi}^{2}=5.552, \mathrm{P}=0.018\right)$ [7]. This study concluded that AR expression was biologically related to TNBC characteristics and behavior which played a further role in endocrinotherapy and prognostic prediction [7]. 
South American Journal of Clinical Research

Volume 3, Issue 1, 2016

Evidence 7: In the study performed by Rakha, E.A., 1726 cases of invasive breast carcinomas were informative for the 3 markers (estrogen receptor, progesterone receptor, and HER2) out of which $282(16.3 \%)$ showed a triple-negative phenotype (estrogen receptornegative, progesterone receptor-negative, and HER2-negative) [9]. Interestingly, in this series of triple-negative breast cancer, basal phenotype as defined by the expression of CK5/6 and/or CK14 in 10\% of tumor cells was detected in 157 (55.7\%) cases [9]. It was also found that basal phenotype, within the triple-negative tumor series, was associated with negative lymph node disease (67\% compared with $58 \%$ in the non-basal phenotype triple-negative tumors), development of distant metastasis, and recurrence; They found that in the poor Nottingham Prognostic Index group (all were lymph node-positive, grade 3 tumors, and sized $1.5 \mathrm{~cm} ; 227$ cases after exclusion of 22 cases of grade 2 or size $<1.5 \mathrm{~cm}$ ) triple negative tumors were associated with shorter overall survival and disease free interval (log-rank $1 / 46.9$, P 1/4.008 and log-rank 1/4 5.99, P 1/4.014) [9]. As a result, it was observed that Triple Negative phenotype was associated with larger size, grade III tumors, pushing margin $\left(X^{2}=6.7\right.$, $\mathrm{P}=0.009$ ), development of recurrence and distant metastasis and poor Nottingham Prognostic index $\left(\mathrm{X}^{2}=112.6, \mathrm{P}<0.001\right)$. Moreover, higher grade was linked to negative E-cadherin expression $\left(\mathrm{X}^{2}=6.4, \mathrm{P}=0.04\right)$ and positive $\mathrm{P}$-cadherin expression $\left(\mathrm{X}^{2}=18.3, \mathrm{P}<0.001\right)$ and p53 $\left(\mathrm{X}^{2}=11.2, \mathrm{P}=0.004\right)$ [9].

Evidence 8: In a study conducted by Garay, J.P., and Park, B.H., they demonstrated that there were some breast cancer cell lines that expressed AR as the sole individual sex hormone receptor [3]. Out of them, one was MDA-MB-453 breast cancer cell line that was observed and expressed in AR (+) and ER (-) status [3]. They conducted an experiment on lab created non-tumorigenic breast cell line, in which they identified that p21 was an important molecule that was required for AR signaling through the MAP Kinase pathway [3].

Evidence 9: A study by Moinfar et al., examined the sex hormone receptor status and HER2 amplification in various grades of carcinoma in situ and invasive cancer [3]. According to them, as the tumor grade progressed from 1 to 3, AR expression started declining from $95 \%$ to $76 \%$ in DCIS and $88 \%$ to $47 \%$ in invasive carcinoma [3]. In the same analysis they also found that ER expression declined from $100 \%$ to $8 \%$ in DCIS and to $9.5 \%$ in invasive carcinoma, as the tumor grade increased [3]. Moreover, they also observed the fact that as the tumor size increased, the HER2 expression also increased from $0 \%$ in grade I to $84 \%$ and $42 \%$ in grade 3 DCIS and invasive carcinoma respectively [3].

Evidence 10: In a study conducted by Gucalp, A., et al., it was observed that the Triple Negative Breast Cancer Metastatic breast cancer patients might get benefitted by the oral administration of Bicalutamide at the dosage of $150 \mathrm{mg} /$ day when followed for 12 weeks [4]. This study was a Phase II, open-label, multicenter, single arm study in which out of enrolled 424 patients, $12 \%$, that was 51 number was found to be ER (-)/PgR (-) that exhibited molecular apocrine or luminal Androgen Receptor [4]. Out of 51 selected patients, only 26 numbers of patients were found to be eligible for the study [4]. As a result, it was observed that Clinical Benefit Rate of $19 \%$ was noted with Bicalutamide that showed proof of principle for the efficacy of minimally toxic androgen blockade [4].

Evidence 11: In a Phase III, double-blinded, placebo-controlled study conducted by Shipley, W.U., et al., it was evaluated that if the anti-androgen therapy was combined with Radiotherapy in Prostate cancer patients, the overall survival of the patients might improve [11]. In their study, 771 patients were found to be eligible to participate out of which 387 patients were enrolled for Radiotherapy + Anti-androgen therapy, and 383 patients were enrolled for Radiotherapy alone [11]. On a follow-up of 7 years, it was observed that Overall survival rate improved accounting 91\% for Radiotherapy + Anti-Androgen Therapy, and 86\% for Radiotherapy alone [11]. Hence, it could be predicted that the addition of Bicalutamide $150 \mathrm{mg}$ daily during and after Radiotherapy could significantly improve the overall survival of the patient suffering from prostate cancer [11]. 


\section{Clinical trials pending}

Following are the awaited high methodology quality Prospective data for evaluating safety and efficacy of Bicalutamide (Anti-Androgen) for the treatment of triple negative, androgen receptor positive breast cancer.

1. NCT02348281: Sponsored by Fudan University, China [14]. The study is Prospective, open-label, Interventional, single-centered, Phase II study to evaluate the efficacy and safety of Bicalutamide as a treatment in androgen receptor (AR) positive metastatic triple negative breast cancer and high clinical benefit rate and a good safety profile in postmenopausal female patients who underwent at least one Chemotherapy regimen for metastatic disease [14].

2. NCT02697032: Sponsored by University Medical Center Groningen, The Netherlands [15]. The Study is Interventional, open-label, single-arm Phase II study to evaluate whether non-invasive in vivo imaging by $18 \mathrm{~F}$ - fluoro - dihydrotestosterone positron emission tomography (FDHT-PET) for Androgen receptor presence in metastatic breast cancer postmenopausal female patients with AR (+) and HER2 (-) tumor can be used to predict early treatment response and optimal dosing of the anti - androgen drug Bicalutamide [15].

3. NCT02353988: Sponsored by Jinling Hospital, China [16]. This study is Interventional, Multicenter, Randomized, Phase II, open-label in female patients with AR (+) Metastatic Triple Negative Breast Cancer to evaluate the anti-tumor activity and safety of Bicalutamide administered orally daily to the patients [16].

4. NCT00468715: Sponsored by Memorial Sloan Kettering Cancer Center [17]. This study is Interventional, open-label, Multicenter, Phase II study to evaluate the anti-tumor activity and safety of Bicalutamide administered orally daily to female patients with ER ()/PR (-)/AR (+) metastatic breast cancer [17].

5. NCT02605486: Sponsored by Memorial Sloan Kettering Cancer Center [18]. This study is Interventional, Open-label, Phase I/II Trial to test the safety and effectiveness of palbociclib with Bicalutamide for the treatment of Triple Negative AR (+) breast cancer females [18].

\section{Conclusion}

The methodology being adopted for the relevant literature review, searching the databases like, PUBMED, Cochrane Library, MEDLINE, EMBASE, Clinical trials.gov was utilized. From the various literature reviewed systematically, it can thus be concluded that Bicalutamide could be used as a competitive antagonist that allowed Androgen Receptor to bind to the DNA. Further, it can also be said that Androgen Receptor expression appeared more frequently in ER (-) and HER2 (-) overexpressing tumors. As a conclusion, Androgen Receptor is now emerging as a useful prognostic marker for diagnosis of breast cancer subtype and new targeted therapeutics followed by proper and timely diagnosis of the underlying disease. Further, AR/ER ratio might play a critical role in influencing tumor biology and response to traditional endocrine therapy. The results of in vitro studies by different authors have suggested that Androgens acted as a growth-promoting factor indicating that presence of Androgen Receptors can be associated with better diagnosis and increased disease-free overall survival of the Triple negative Breast cancer post-menopausal female suffering. Currently, the traditional diagnosis methods such as IHC and FISH are being employed to detect and confirm the nodal status, histological grade of the tumor, as well as, ER, PR and HER2 status. Proper evaluation of these biomarkers is of utmost importance in order to elucidate the response of targeted therapies. Multiple studies also indicated the efficacy of Bicalutamide in postmenopausal setting. Further, AR expression decreased with increase in the tumor grade, however, the ER expression level increased. On the other hand, a clear and comprehensive understanding of the mechanism of Androgen Receptor signaling in the breast carcinoma is yet to be discovered. 
South American Journal of Clinical Research

Volume 3, Issue 1, 2016

From the case study, it can be analyzed that Triple Negative Breast Cancer patient is sensitive to Chemotherapy. However, the patient tolerated the traditional anti-androgen, Bicalutamide well. The overall survival of the said TNBC metastatic patient was diagnosed to be more than 5 years. Despite of some side effects associated with the drug intake like, fatigue, limbs pain, all other blood reports examined were diagnosed within the normal range, this fact somehow pointed in a direction that by considering all the biological aspects of the patient, the drug Bicalutamide is safe and efficacious up to certain extent. On the whole, it can be summarized that the Anti-Androgen drug, Bicalutamide could be more effective in TNBC metastatic post-menopausal patients who have underwent at least one chemotherapy cycle and one Radiotherapy cycle.

After doing the critique, there were certain questions that need further insights. Research is required to investigate the relationship between Anti-Androgens and their action of blocking Estrogens. Secondly, to identify the correlation between Androgen Receptor and HER2/neu, correlation between Androgen status and Ki-67 expression, correlation between Androgen Receptor and menopausal status in Triple negative metastatic breast cancer patients. Therefore, it is very essential to elucidate the functional role of Androgen Receptor in breast cancer. Thus, Androgen receptor could be said to be expressed in approximately $80 \%$ of primary breast cancer in which Androgen Receptor was believed to be co-localized with Estrogen Receptor and Progesterone Receptor in breast epithelial cells which was not detected in mammary stroma and the myoepithelium.

\section{References}

[1.] ClinicalTrials.gov [Internet]. Xichun, Hu.: Fudan University (China). 2015 January 18- Identifier NCT02348281, Bicalutamide as A Treatment in AR-positive Metastatic Triple-Negative Breast Cancer (mTNBC) Patients; Accessed on: 2016 March 19; Available from:

https:/clinicaltrials.gov/ct2/show/record/NCT02348281?term=androgen+receptor+and+bicalutamide\& rank $=1$.

[2.] ClinicalTrials.gov [Internet]. Schroder, C.P.: University Medical Center Groningen (The Netherlands). 2015 August 25- Identifier NCT02697032, FDHT PET and Bicalutamide in Metastatic Breast Cancer; Accessed on: 2016 March 19; Available from:

https:/clinicaltrials.gov/ct2/show/record/NCT02697032?term=androgen+receptor+and+bicalutamide\& rank=2.

[3.] ClinicalTrials.gov [Internet]. Guan, X. : Jinling Hospital (China). 2015 January 26- Identifier NCT02353988, AR-inhibitor Bicalutamide in Treating Patients With TNBC (Arbre); Accessed on: 2016 March 19; Available from:

https:/clinicaltrials.gov/ct2/show/record/NCT02353988?term=androgen+receptor+and+bicalutamide\& rank=4.

[4.] ClinicalTrials.gov [Internet]. Memorial Sloan Kettering Cancer Center (United States). 2007 May 2- Identifier NCT00468715, Bicalutamide in Treating Patients With Metastatic Breast Cancer; Accessed on: 2016 March 19; Available from:

https:/clinicaltrials.gov/ct2/show/study/NCT00468715?term=androgen+receptor+and+bicalutamide\&r ank $=7$.

[5.] ClinicalTrials.gov [Internet]. Memorial Sloan Kettering Cancer Center (United States). 2015 November 12- Identifier NCT02605486, Palbociclib in Combination With Bicalutamide for the Treatment of AR(+) Metastatic Breast Cancer (MBC); Accessed on: 2016 March 19; Available from:

https:/clinicaltrials.gov/ct2/show/study/NCT02605486?term=androgen+receptor+and+bicalutamide\&r ank=14.

[6.] Cochrane, D.R., Bernales, S., Jacobsen, B.M., Cittelly, D.M., Howe, E.N., D’Amato, N.C., Spoelstra, N.S., Edgerton, S.M., Jean, A., Guerrero, J., Gómez, F., Medicherla, S., Alfaro, I.E., McCullagh, E., Jedlicka, P., Torkko, K.C., Thor, A.D., Elias, A.D., Protter, A.A., and Riche, J.K. (2014). Role of the androgen receptor in breast cancer and preclinical analysis of enzalutamide. Breast Cancer Research, 16, R7. http://doi:10.1186/bcr3599. 


\section{South American Journal of Clinical Research}

Volume 3, Issue 1, 2016

[7.] Doane, A.S., Danso, M, Lal, P., Donaton, M., Zhang L., Hudis, C., and Gerald, W.L. (2006). An estrogen receptor-negative breast cancer subset characterized by a hormonally regulated transcriptional program and response to androgen. Oncogene, 25, 3994-4008. http://doi:10.1038/sj.onc.1209415.

[8.] Garay, J. P., \& Park, B. H. (2012). Androgen receptor as a targeted therapy for breast cancer. American Journal of Cancer Research, 2(4), 434-445. Available at:

http://www.ncbi.nlm.nih.gov/pmc/articles/PMC3410582/.

[9.] Gucalp, A., Tolaney, S., Isakoff, S.J., Ingle, J.N., Liu, M.C., Carey, L.A., Blackwell, K.., Rugo, H., Nabell, L., Forero, A., Stearns, V., Doane, A.S., Danso, M., Moynahan, M.E., Momen, L.F., Gonzalez, J.M., Akhtar, A., Giri, D.D., Patil, S., Feigin, K.N., Hudis, C.A., and Traina, T.A. (2013). Phase II trial of bicalutamide in patients with androgen receptor-positive, estrogen receptor-negative metastatic Breast Cancer. Clin Cancer Res, 19, 5505-12. http://doi:10.1158/1078-0432.CCR-12-3327.

[10.] Kotsopoulos, J., Narod, S.A. (2012). Androgens and breast cancer. Steroids, 77, 1-9. http://:10.1016/j.steroids.2011.10.002.

[11.] Lehmann, B. D., Bauer, J. A., Chen, X., Sanders, M. E., Chakravarthy, A. B., Shyr, Y., \& Pietenpol, J. A. (2011). Identification of human triple-negative breast cancer subtypes and preclinical models for selection of targeted therapies. The Journal of Clinical Investigation, 121(7), 2750-2767. http://doi.org/10.1172/JCI45014.

[12.] Luo, X., Shi, Y.X., Li, Z.M., Jiang, W.Q. (2010). Expression and clinical significance of androgen receptor in triple negative breast cancer. Chin J Cancer, 29(6), 585-90. Available at: www.cjcsysu.cn.

[13.] Min Ni, M., Yiwen Chen, Y., Elgene Lim, E., Hallie Wimberly, H., Shannon, T., Bailey, S.T., Yuuki Imai, Y., David, L., Rimm, D.L., X. Shirley Liu, X.S., Myles Brown, M. (2011). Targeting Androgen Receptor in Estrogen Receptor-Negative Breast Cancer. Cancer Cell, 20, 119-131. http://dx.doi.org/10.1016/j.ccr.2011.05.026.

[14.] Rakha, E.A., El-Sayed, M.E., Green, A.R., Lee, A.H., Robertson, J.F., Ellis, I.O. (2007). Prognostic markers in triple-negative breast cancer. Cancer, 109(1):25-32. http://doi:10.1002/cncr.22381.

[15.] Shibahara, Y., Miki, Y., Sakurada, C., Uchida, K.., Hata, S., McNamara, K.., Yoda, T., Takagi, K.., Nakamura, Y., Suzuki, T., Ishida, T., Ohuchi, N., Sasano, H. (2013). Androgen and androgenmetabolizing enzymes in metastasized lymph nodes of breast cancer. Human Pathology, 44, 2338 2345. http://dx.doi.org/10.1016/j.humpath.2013.04.021.

[16.] Shipley, W.U., Hunt, D., Lukka, H.R., Major, P., Heney, N.M., Grignon, D., Patel, M., Bahary, J., Lawton, C.A., and Sandler, H.M. (2011). Journal of Clinical Oncology, 29 (7), 1. Available at: http://hwmaint.meeting.ascopubs.org/cgi/content/abstract/29/7_supp1/1.

[17.] Srikanth, M.V., Janaki Ram, B., Senthil Rajan, D.S., Adinarayana, G. and Ramana Murthy, K.V. (2013). Dissolution rate enhancement of bicalutamide by adsorption process. African Journal of Pharmacy and Pharmacology, 7 (21), 1357-1362. http://doi10.5897/AJPP10.073.

[18.] U.S. National Library of Medicine. (Updated 2016, February 24). Bicalutamide. Retrieved March 19, 2016, from Medline Plus:

https://www.nlm.nih.gov/medlineplus/druginfo/meds/a697047.html. 